\title{
Stability, Inter-Strand Contact Resistance and AC Losses in YBCO Roebel Cables
}

\author{
M. Majoros, M. D. Sumption, E. W. Collings and N. J. Long
}

\begin{abstract}
A de transport current was applied to the strands of a Roebel cable at $77 \mathrm{~K}$ in liquid nitrogen bath. The inter-strand contact resistance was measured. It was modified either by applying a pressure on the cable at $77 \mathrm{~K}$ in liquid nitrogen bath or using different soldering patterns between the strands of the cable. Magnetization ac losses were measured in frequency range $50-200 \mathrm{~Hz}$ in applied magnetic field $4-70 \mathrm{mT}$ perpendicular to the broader face of the cable to test the inter-strand contact resistance effect. High stability and very low level of coupling losses was observed in the cables even with the lowest interstrand resistances.
\end{abstract}

Index Terms-Current sharing, Roebel cable, stability, superconductor, YBCO.

\section{INTRODUCTION}

Y BCO coated conductor technology has brought high temperature superconducting magnets and machines much closer to reality. The dc performance of YBCO tapes has been greatly improved by enhancing the in-field critical current density through the addition of artificial pinning centers [1]. However, their performance in a time-varying magnetic fields is less satisfactory due to the ac losses generated. A significant reduction in magnetic ac loss is a prerequisite for the use of coated conductor tapes in large scale applications. A promising design of a low ac loss cable is based on the classical Roebel cable concept, in which the component strands are magnetically decoupled without twisting the conductor (clarify e.g. [2] and references therein). If YBCO tapes are assembled into Roebel cables, hysteresis and stabilizer eddy-current losses of the individual strands are still present, but an additional loss component arises from coupling currents that circulate along entire strands and across the inter-strand contact regions [3], [4]. At high field ramp rates the coupling losses may cause instability and premature quenching, or ramp rate sensitivity [5]. The coupling can be eliminated by full strand insulation. However, implementation of this solution would prevent current sharing between strands and hence would lead to a lower stability. Therefore one of the goals of modern cable design is to modify the inter-strand contact resistance in such a way that the conflicting

Manuscript received 15 July 2013.

M. Majoros, M. D. Sumption, and E. W. Collings are with the Center for Superconducting and Magnetic Materials (CSMM), Department of Materials Science and Engineering, The Ohio State University, Columbus, OH 43210, USA (phone: 614-688-5344; fax: 614-292-1537; e-mail: majoros.2@osu.edu).

N. J. Long is with the Industrial Research Limited, Lower Hutt 5040, New Zealand requirements of low coupling losses and stability are simultaneously satisfied.

The purpose of the present work is to investigate the influence of different methods, such as pressure or soldering, on interstrand contact resistance and magnetization ac losses in YBCO Roebel cables.

\section{EXPERIMENTAL}

\section{A. Samples}

All the experiments were performed on a Roebel cable with the parameters given in Table 1. Three different modifications of the cable were studied. They are denoted as Sample 1, Sample 2 and Sample 3 (Table 2). Although a resistivenetwork model of the Roebel cable has not been explicitly offered, a useful insight into inter-strand contact resistance can be obtained by considering the Roebel cable as a topologically transformed Rutherford cable. The wide side of the Rutherford cable is equivalent to the narrow side of the Roebel cable and vice versa. Two main contact resistances exist in such cables a contact resistance $R_{c}$ between crossing strands and a contact resistance $R_{a}$ between adjacent strands (clarify [6], Chapter 4). In exploring ways to reduce $R_{a}$ - (i) a massive steel plates were used to apply pressure to the cable (Sample 1) (ii) a thin strip of tin was laid along the broad face of the cable (Sample 2), (iii) copper shunts were intermittently soldered across the broad face of the cable (Sample 3). In exploring the effects of these procedures on $R_{a}$ measurements were made using the direct current-voltage $(I-V)$ technique at $77 \mathrm{~K}$ in liquid nitrogen bath. In this method two strands on opposite edges of a cable piece are connected to a current supply, while the voltage is measured over any combination of strands [6] (Chapter 4). This is schematically shown in Fig. 1. A measured voltage is symmetric around a peak obtained when measured between the edge and the central strand. The shape of the $I$ - $V$ curve is a good indication of the ratio between $R_{a}$ and $R_{c}$. A shallow $I-V$ curve with small peak is observed for $R_{c}$ $\leq R_{a}$ while a sharp dependence with a strong peak is observed for $R_{a}<<R_{c}$ [6] (Chapter 4).

Numerical calculations of the peak voltage $U_{e e}$ over the two edges of the cable (Fig. 1) result in the following analytical expressions [4] (Chapter 4)

$$
U_{e e}=\left(\frac{R_{a} R_{c}}{R_{a}+R_{c}}\right) \frac{L_{p, s}}{l_{c a b} N_{s}} I
$$

for $R_{c} \leq R_{a}$ and $\rho_{s} \rightarrow 0$ and 


$$
U_{e e}=\frac{L_{p, s} R_{a}}{2 l_{c a b} \sqrt{N_{s}}} I
$$

for $R_{a}<<R_{c}$ and $\rho_{s} \rightarrow 0$. Here $I$ is the current, $L_{p, s}$ is the twist pitch, $l_{c a b}$ is the cable length, $N_{s}$ is the number of strands in the cable and $\rho_{\mathrm{s}}$ is the strand resistivity. Using these equations the contact resistances $R_{a}$ and $R_{c}$ can be estimated from the measured maxima of the $I-V$ curves. The photos of measured samples with wiring patterns are shown in Fig. 2 - 4.

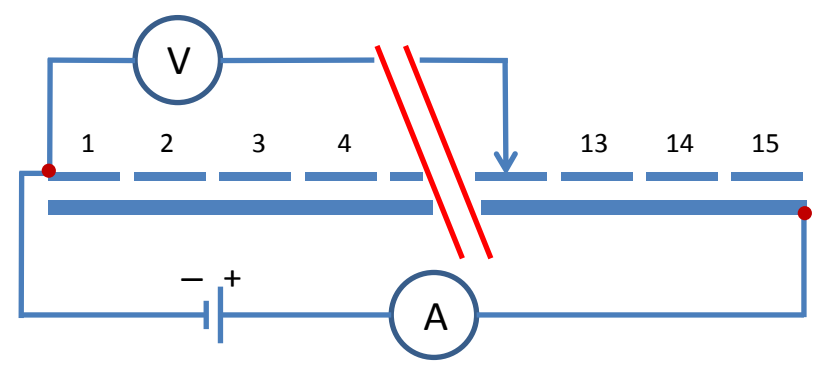

Fig. 1: Illustration of the $I-V$ method. Two strands on opposite edges of a cable piece are connected to a current supply and the voltage is measured over any combination of the strands.

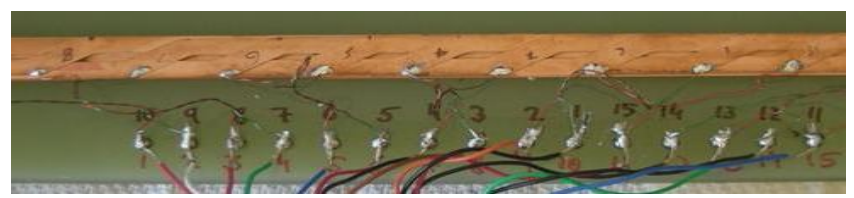

Fig. 2: Sample 1 -wiring of the cable.

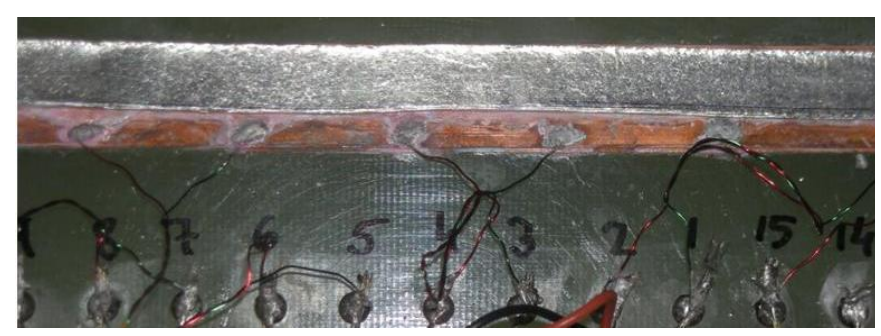

Fig. 3: Sample $2-\mathrm{Sn}$ foil (8 $\mathrm{mm}$ wide, $1 \mathrm{~mm}$ thick) position and cable wiring.

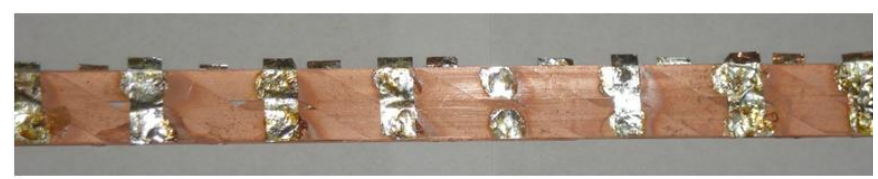

Fig. 4: Sample 3 - soldering pattern. Width of the copper shunts (strips) is $5 \mathrm{~mm}$ and thickness $0.1 \mathrm{~mm}$.

\section{B. Measuring procedures}

In the $I-V$ curve method we used a high quality HP6634 A $(1 \mathrm{~A}-100 \mathrm{~V}) \mathrm{DC}$ power source to supply the current. The voltage was measured by a high-sensitivity Keithley 2182A nanovoltmeter. All the measurements were made at applied current of 0.5 A or $1 \mathrm{~A}$. We measured a voltage between one potential tap fixed (tap 1, Fig. 1) and the other one
TABLE I

PARAMETERS OF THE ROEBEL CABLE

\begin{tabular}{lc}
\hline \multicolumn{1}{c}{ Quantity } \\
\\
\hline Cable width (mm) & 13 \\
Cable thickness $(\mathrm{mm})$ & 1.5 \\
Cable twist pitch $(\mathrm{cm})$ & 30 \\
Cable length $(\mathrm{cm})$ & 30 \\
Number of tapes in the & 15 \\
cable & \\
Tape width (mm) & 5 \\
Cable $\mathrm{I}_{\mathrm{c}}(77.3 \mathrm{~K}, \mathrm{self}-$ & 1537.5 \\
field) $(\mathrm{A})$ & \\
\hline
\end{tabular}

TABLE II

SAMPLES PREPARED FROM THE ROEBEL CABLE

\begin{tabular}{cl}
\hline \hline Sample & \\
\hline 1 & as received cable \\
2 & cable with $\mathrm{Sn}$ foil on its top \\
3 & cable with soldered $\mathrm{Cu}$ shunts \\
\hline
\end{tabular}

positioned along the cable length (tap numbers $n=2,3 \ldots$ etc, Fig. 1). These voltages were denoted as $U_{1-n}$ and plotted against $n$ (Fig. $5-8$ ).

Magnetization ac losses were measured using an $\mathrm{M}(\mathrm{H})$ loop method. The magnetic field was generated by a race-track copper magnet cooled in liquid nitrogen bath. The magnet was equipped with a system of pick-up and compensation coils. Compensated voltages from the system of these coils were measured by a sensitive digital oscilloscope. Area of the hysteresis loop measured by the oscilloscope was calculated and ac loss per cycle plotted in dependence on applied magnetic field amplitude. Measurements of ac losses were performed in the frequency range $50-200 \mathrm{~Hz}$ and in magnetic field amplitude range $4-70 \mathrm{mT}$.

To generate pressure massive steel plates were used. The weight of a single plate was $55.7 \mathrm{~N}$ resulting in a pressure of 2 $\mathrm{kPa}$ per plate. Up to four plates were used with the maximum pressure of $8 \mathrm{kPa}$.

\section{RESULTS AND DISCUSSION}

\section{A. Pressure Effects on I-V curves}

The results of $I$ - $V$ curves for Sample 1 measured at different pressures are shown in Fig. 5. It is seen that applied pressure has a significant effect on cable inter-strand resistance. At 8 $\mathrm{kPa}$ the strand resistance decreased nearly 5-times. Maximum inter-strand resistance without pressure is $0.47 \Omega$ and with a pressure of $8 \mathrm{kPa}$ it is $0.105 \Omega$. Comparing these results with theoretical calculations in [6] (Chapter 4) one can conclude that the shape of $I-V$ curve with no pressure is indicative of $R_{a}$ $\ll R_{c}$ while the curves under pressure rather indicate $R_{a} \approx R_{c}$.

\section{B. Effects of Tin Foil on I-V curves}

The effect of tin foil on the inter-strand resistance of Sample 2 is summarized in Fig. 6. In this case application of pressure is much more beneficial than in case of Sample 1. The fact that the tin material is soft is helpful in filling the space due to the surface roughness of the stabilizing copper coatings of the 
strands in the Roebel cable. The lowest inter-strand resistance obtained at $8 \mathrm{kPa}$ by this method is $31.5 \mathrm{~m} \Omega$. Without the tin foil the inter-strand resistance (Sample 1 ) was $0.108 \Omega$ at 8 $\mathrm{kPa}$ (Fig. 7). So the presence of the tin foil decreases the interstrand resistance by a factor of 3.43 at $8 \mathrm{kPa}$. Again comparing these results with theoretical calculations in [6] (Chapter 4) one can conclude that these $I-V$ are indicative of $R_{a} \ll R_{c}$.

\section{Effect of Soldered Copper shunts on I-V curves}

Effect of soldered copper shunts on inter-strand resistance of the Roebel cable (Sample 3) is shown in Fig. 8. The observed small negative voltages for taps 2 and 15 are due to thermal forces. The soldered copper shunts have a huge effect on lowering the inter-strand resistance. Maximum inter-strand resistance achieved in this configuration is $100 \mu \Omega$.

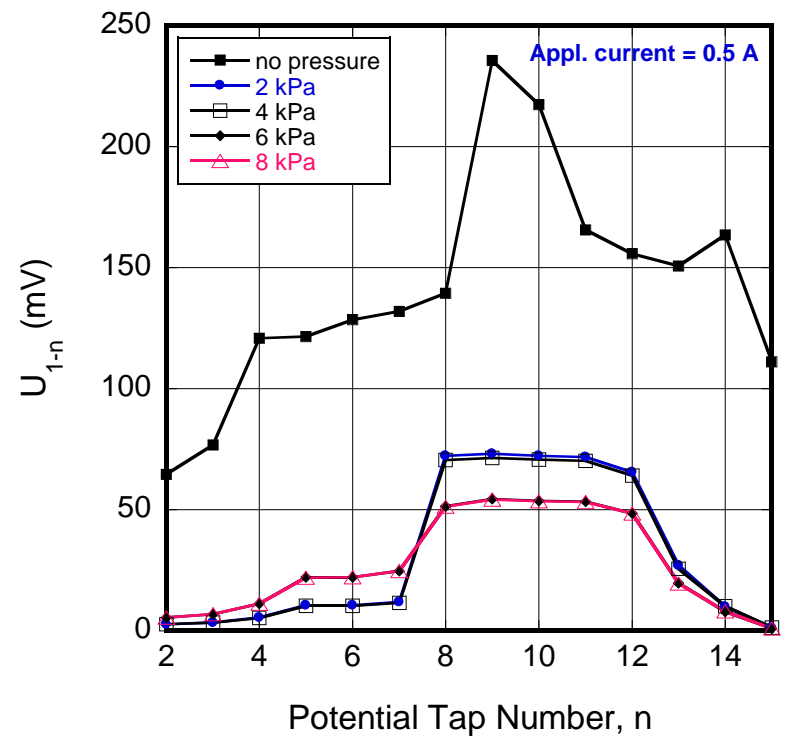

Fig. 5: Sample 1 - effect of pressure on $I$ - $V$ curves $(I=0.5$ A). The shape of I-V curve with no pressure is indicative of $R_{a}$ $\ll R_{c}$ while the curves under pressure rather indicate $R_{a} \approx R_{c}$.

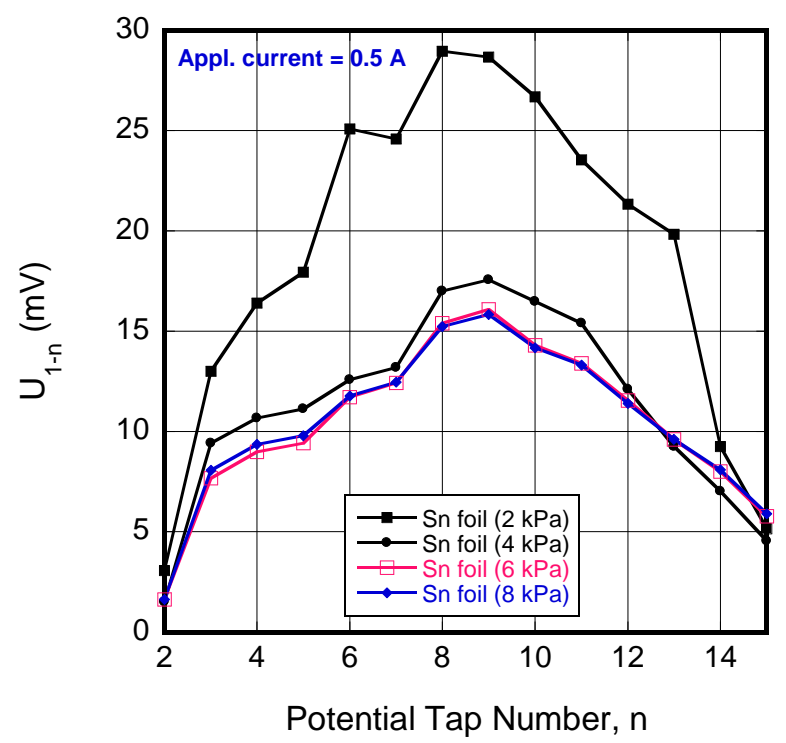

Fig. 6: Sample 2 - effect of pressure on $I-V$ curves $(I=0.5$ A) (indicative of $R_{a} \ll R_{c}$ ).
This value is 315-times lower than the maximum inter-strand resistance achieved by the tin foil at $8 \mathrm{kPa}$ (Sample 2) (Fig. 6, 7) and 1050 times lower than the maximum inter-strand resistance of Sample 1 at $8 \mathrm{kPa}$. Comparisons with theoretical calculations in [6] (Chapter 4) indicate that $R_{a} \ll R_{c}$. The main results on $I-V$ curve measurements for all three samples are summarized in Table 3.

\section{A. Effects of Soldered Copper Shunts on AC Losses}

Magnetization ac losses were measured on Sample 3 in frequency range $50-200 \mathrm{~Hz}$. The results are summarized in Fig. 9. Despite quite a low maximum inter-strand resistance of Sample 3 (of $100 \mu \Omega$ ) the frequency dependence of the energy loss per cycle is rather weak.

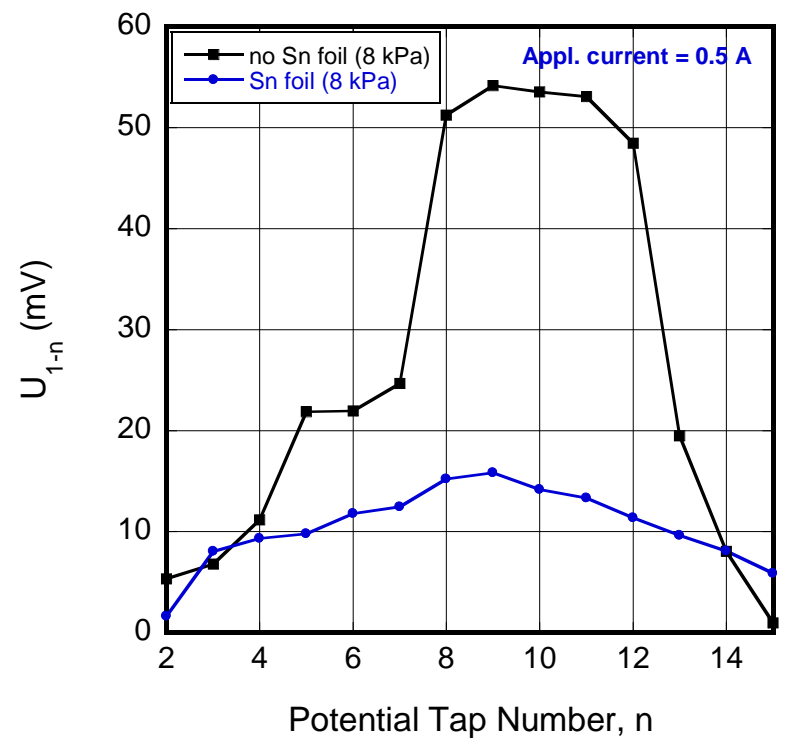

Fig. 7: Sample 2 - effect of pressure on $I$ - $V$ curves (a comparison with no Sn curve) $(I=0.5 \mathrm{~A})$.

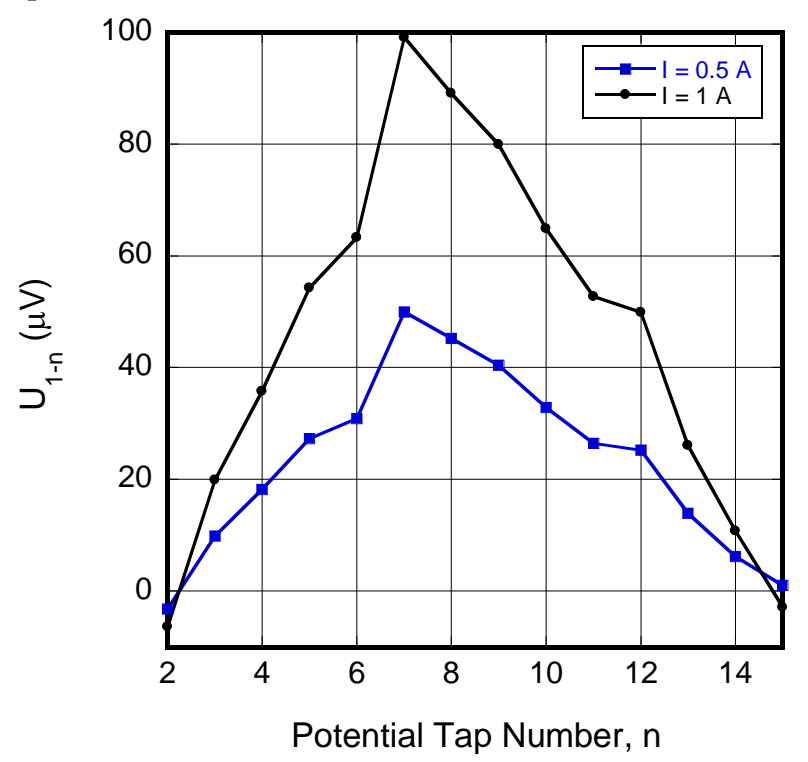

Fig. 8: Sample 3 - effect of current on $I$ - $V$ curves $(I=0.5 \mathrm{~A}$ and $1 \mathrm{~A})$. The shape of $I-V$ curves is indicative of $R_{a} \ll R_{c}$.

This is in accordance with the results published in [4]. In 
TABLE III

MAXIMUM INTER-STRAND RESISTANCE OF SAMPLE 1, 2 AND 3

\begin{tabular}{ccc}
\hline \hline \multirow{2}{*}{ Sample } & Pressure $(\mathrm{kPa})$ & $\begin{array}{c}\text { Maximum inter-strand resistance } \\
(\mathrm{m} \Omega)\end{array}$ \\
\hline 1 & 8 & 105.0 \\
2 & 8 & 31.5 \\
3 & - & 0.1 \\
\hline
\end{tabular}

low field region $(<0.01 \mathrm{~T})$ ac losses show visible frequency dependence (see Fig. 9, inset) with maximum around $100 \mathrm{~Hz}$ (a typical "finger print" of coupling loss). At higher fields the magnetic field starts to penetrate the superconducting YBCO film and ac losses increase in a non-linear way. However, qualitatively their frequency dependence shown in the inset of Fig. 9 is retained in the whole range of magnetic field amplitudes (invisible in log-log plots in Fig. 9). Observation of this kind of weakly frequency dependent ac loss is in agreement with the critical state model [7]. Based on these results one can conclude that the maximum inter-strand resistance of $100 \mu \Omega$ is still quite high to allow significant coupling currents to circulate along the entire strands and across the inter-strand contact regions. A qualitative understanding of this problem is possible based on a theoretical work by Takacs [8] - [10]. In that work a problem

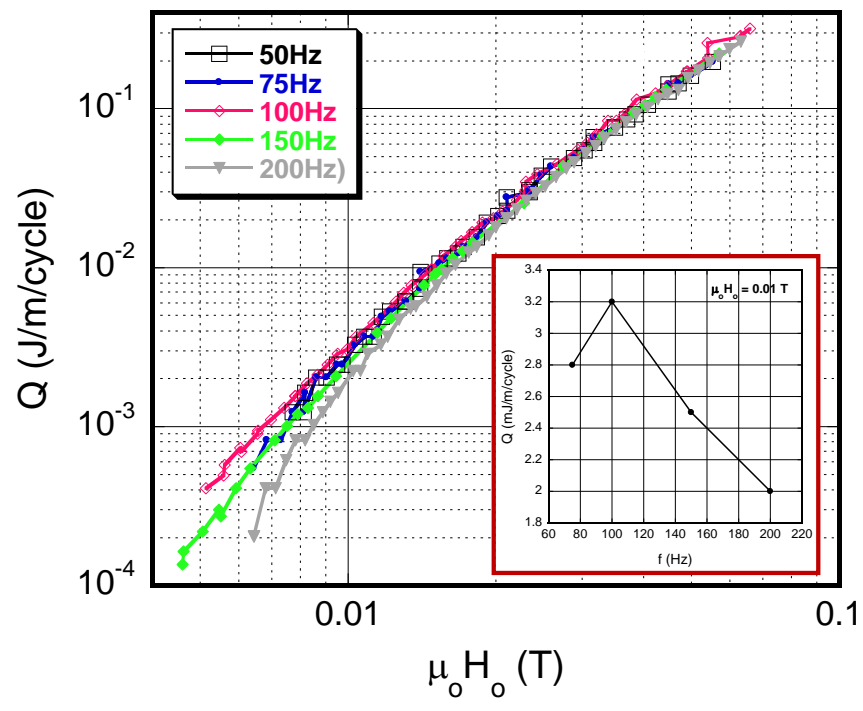

Fig. 9: Sample 3 - magnetization energy loss per cycle and per $1 \mathrm{~m}$ cable length vs. applied magnetic field amplitude at different frequencies (log-log scales). The inset shows the energy loss vs. frequency at magnetic field amplitude of 0.01 $\mathrm{T}$ (linear scales).

of acceptable coupling losses in striated coated conductors or twisted cables, ensuring current sharing between superconducting filaments was analyzed. Even though in that work only a problem of normal segments which are coated above the straight striated superconducting stripes [8] was considered, the results can be, at least qualitatively, applied also to ac losses in Roebel cables. From the theory [8] - [10] for the hysteresis loss $\mathrm{W}_{\mathrm{h}}$ we have

$$
\frac{W_{h}}{l}=N w^{2} f J_{c} B_{m}
$$

Here $l$ is the length of the sample, $N$ - number of strands, $w$ - strand width, $f$ - frequency, $J_{c}$ - the sheath critical current density (equal to $I_{c} / w$ ) and $B_{m}$ is the applied magnetic field amplitude. Taking the critical current of our Roebel cable from Table 1 we get $J_{c}=2.05 \times 10^{4} \mathrm{~A} / \mathrm{m}$. For $B_{m}=0.06 \mathrm{~T}$ from (3) we get $W_{h} / l / f=0.455 \mathrm{~J} / \mathrm{m} /$ cycle. This value is quite close to the measured value (Fig. 9) of $0.25 \mathrm{~J} / \mathrm{m} /$ cycle on our Sample 3. So the model describes ac loss in Roebel cables with a precision of about $50 \%$. For the ratio of the coupling loss $W_{c}$ to the hysteresis loss $W_{h}$ from the theory [8] we have

$$
\frac{W_{c}}{W_{h}}=\frac{4 \pi^{2} B_{m} \text { fhbd }^{3}}{9 \rho N w^{2} J_{c} l_{0}}
$$

Here $h$ is the normal plating thickness, $b$ - cable width, $d-$ normal plating width, $\rho$ - effective resistivity (its value depends on the structure of the normal segments (shunts)), $l_{0}$ is the normal plating (shunts) separation [6]. Taking the maximum inter-strand resistance of $100 \mu \Omega$ (Fig. 8) from our experiments, using (2) and the geometry of Sample 3 (Table 1) we get $\rho=5 \times 10^{-7} \Omega \mathrm{m}$. Alternatively, we can derive the effective resistivity using an expression for the time constant of coupling currents [11] $\tau=\mu_{o} L_{p, s}^{2} /\left(8 \pi^{2} \rho\right)$. Then from the condition of the coupling loss maximum $\omega \tau=1$, where $\omega$ is the circular frequency equal to $2 \pi f$, the resistivity $\rho$ can be calculated. Using $f=100 \mathrm{~Hz}$ as a frequency at which the maximum ac loss was observed and the twist pitch $L_{p, s}$ of the cable (Table 1) we get $\rho=10^{-7} \Omega \mathrm{m}$. This value is comparable with the value of $5 \times 10^{-7} \Omega \mathrm{m}$ obtained from the $I-V$ curve measurements using equation (2). Inserting the value $\rho=10^{-7}$ $\Omega \mathrm{m}$ in equation (4) we get $W_{c} / W_{h}=5.2 \times 10^{-4}$ at $f=200 \mathrm{~Hz}$ for magnetic field amplitude of $0.06 \mathrm{~T}$. This means that coupling losses are negligibly small compared with the hysteresis loss as observed experimentally. On the other hand the maximum inter-strand resistance of $100 \mu \Omega$ might be sufficiently low to enable a significant current sharing between the strands in the Roebel cables. The results on this will be published elsewhere.

\section{CONCLUSION}

In an effort of aiming current sharing between the strands in Roebel cables we studied experimentally the effect of different kinds of inter-strand connections on inter-strand resistances. Just applying a pressure of $8 \mathrm{kPa}$ gives a maximum interstrand resistance of $105 \mathrm{~m} \Omega$. Using a tin foil placed on top of the Roebel cable gives the maximum inter-strand resistance of $31.5 \mathrm{~m} \Omega$ under a pressure of $8 \mathrm{kPa}$. The lowest maximum inter-strand resistance of $0.1 \mathrm{~m} \Omega$ was achieved by soldering copper shunts (strips $5 \mathrm{~mm}$ wide and $0.1 \mathrm{~mm}$ thick). Even such a low inter-strand resistance value does not cause any significant coupling loss increase up to $200 \mathrm{~Hz}$. However, on the other hand it will allow some current sharing between the strands of the Roebel cables. Detailed experimental results on this subject will be published elsewhere.

\section{ACKNOWLEDGMENT}

This work was supported by the U.S. Department of Energy, grant DE-FG02-07ER84913. 


\section{REFERENCES}

[1] P. Mele, K. Matsumoto, T. Horide, A. Ichinose, M. Mukaida, Y. Yoshida, S. Horri and R. Kita, "Ultra-high flux pinning properties of $\mathrm{BaMO}_{3}$-doped $\mathrm{YBa}_{2} \mathrm{Cu}_{3} \mathrm{O}_{7-\mathrm{x}}$ thin films $(\mathrm{M}=\mathrm{Zr}, \mathrm{Sn})$," Supercond. Sci. Technol. vol. 21, p. 032002, 2008.

[2] L. S. Lakshmi, M. P. Staines, R. A. Badcock, N. J. Long, M. Majoros, A. W. Collings and M. D. Sumption, "Frequency dependence of magnetic ac loss in a Roebel cable made of YBCO on a Ni-W substrate," Supercond. Sci. Technol. vol. 23, p. 085009, 2010.

[3] S. Terzieva, M. Vojenciak, E. Pardo, F. Grilli, A. Drechsler, A. Kling, A. Kudymov, F. Gomory and W. Goldacker, "Transport and magnetization ac losses of ROEBEL assembled coated conductor cables: measurements and calculations," Supercond. Sci. Technol. vol. 23, p. 014023, 2010.

[4] M. Vojenciak, F. Grilli, S. Terzieva, W. Goldacker, M. Kovacova and A. Kling, "Effect of self-field on the current distribution in Roebelassembled coated conductor cables," Supercond. Sci. Technol. vol. 24, p. 095002, 2011.

[5] M. D. Sumption, E. W. Collings, R. M. Scanlan, A. Nijhuis, H. H. J. ten Kate, S. W. Kim, M. Wake and T. Shintomi, "Influence of strand surface condition on inter-strand contact resistance and coupling loss in NbTiwound Rutherford cables," Cryogenics vol. 39, pp. 197-208, 1999.

[6] A. P. Verweij, PhD thesis, University of Twente, Enschede, the Netherlands, 1995. http://cern-verweij.web.cern.ch/cern-verweij/

[7] C. P. Bean, "Magnetization of Hard Superconductors," Phys. Rev. Lett. vol. 8, pp.250-253, 1962.

[8] S. Takacs, "Low coupling losses in $\mathrm{YBa} 2 \mathrm{Cu} 3 \mathrm{O} 7$ coated conductors with current sharing between the superconducting stripes," Appl. Phys. Lett. vol. 90, p. 242505, 2007.

[9] S. Takacs, "Acceptable coupling losses in coated conductors at industrial frequencies without twisting the superconducting stripes," J. Appl. Phys. vol. 103, p. 05397, 2008.

[10] S. Takacs, "Acceptable coupling losses in striated coated conductors or twisted cables ensuring current sharing between superconducting filaments," Supercond. Sci. Technol. vol. 26, p. 055022, 2013.

[11] A. V. Gurevich, R. G. Mints, A. L. Rakhmanov, The physics of composite superconductors, Begel House, inc., New York, Wallingford (UK), 1997, pp. 91 - 102. 